\title{
NEPS - NÚCLEO DE EDUCAÇÃO PERMANENTE EM SAÚDE: FERRAMENTA DE GESTÃO DO HOSPITAL DISTRITAL DR. JOSÉ GOMES DA SILVA
}

\section{NEPS - PERMANENT HEALTH EDUCATION CORE: MANAGEMENT TOOL OF DR. JOSÉ GOMES DA SILVA DISTRICT HOSPITAL}

\author{
George Carneiro Diniz \\ Especialista em Gestão em Programas de \\ Saúde da Família pela Universidade Cândido \\ Mendes - UCAM. Curso de Bacharelado em \\ Psicologia pelas Faculdades Integradas de \\ Patos - FIP, Brasil, Brasil. \\ E-mail: george.psic.arte@gmail.com
}

Mayara Araújo Estrela Diniz Pós-Graduando em Ginecologia,Obstetrícia e Neonatologia. Curso de Bacharelado em Enfermagem pela Faculdade Santa Emília de Rodat, Brasil, Brasil.

E-mail: enfmayestrela2010.2@gmail.com

José Marciel Araújo Porcino Especialista em Psicopedagogia Clínica e Institucional, Especialista em Saúde Mental, Curso de Bacharelado em Psicologia e Licenciando Pedagogia pela Universidade Federal da Paraíba-UFPB na modalidade de Educação a Distância-EAD, Brasil, Brasil. E-mail: leicram_ap@hotmail.com

Marcone Torres da Silva

Graduando do Curso de Bacharelado em Enfermagem pela Faculdade Integrada CETE Garanhuns - PE, Brasil, Brasil. E-mail:marconeetorress@gmail.com

Recebido: 20/11/2020 - Aceito: 27/11/2020

\section{Resumo}

Esta pesquisa está relacionada a Educação Permanente em Saúde, tendo a mesma como uma ferramenta de gestão objetivando identificar a percepção dos funcionários do Hospital Distrital Dr. José Gomes da Silva, localizado no município de Itaporanga - PB, no que diz respeito ao trabalho desenvolvido pelo Núcleo de Educação Permanente em Saúde (NEPs) visando o fortalecimento do trabalho multidisciplinar intersetorial a partir de prioridades estabelecidas na construção do Plano de Ação da instituição de saúde. Entretanto, um dos fatores mais relevantes 
diante dessa proposta é a de uma reflexão crítica dos funcionários acerca do desenvolvimento do trabalho realizado. Para isso, a metodologia utilizada trata-se de uma pesquisa de cunho exploratório-descritivo, fazendo uma análise interpretativa de modo documental, buscando informações em documentos, sendo estes fontes primárias. Contudo, espera-se que as atividades aqui dadas como prioridades e propostas sejam levadas a diante e seguidas com o intuito de transformar práticas cotidianas em trabalho e relação envolvendo gestores, trabalhadores e usuários do serviço de saúde, tendo como foco principal um melhor desenvolvimento do serviço e, consequentemente um serviço mais humanizado, levando em consideração o bem estar do usuário.

Palavras-Chave: Educação Permanente; Saúde; Gestão.

\section{Abstract}

This research is related to Permanent Health Education, having it as a management tool aiming to identify the perception of the employees of Dr. José Gomes da Silva District Hospital, located in the municipality of Itaporanga - PB, regarding the work developed by the Center for Permanent Education in Health (NEPs) aiming at strengthening intersectoral multidisciplinary work based on priorities established in the construction of the Action Plan of the health institution. However, one of the most relevant factors in view of this proposal is that of a critical reflection of employees about the development of the work performed. Therefore, the methodology used is an exploratory-descriptive research, making an interpretative analysis in a documentary way, seeking information in documents, these being primary sources. However, it is expected that the activities given here as priorities and proposals will be carried forward and followed with the intention of transforming daily practices into work and relationship involving managers, workers and users of health services, having as main focus a better development of health care. consequently a more humanized service, taking into account the welfare of the user.

Keywords: Permanent Education,; Health; Management.

\section{Introdução}

O Núcleo de Educação Permanente em Saúde (NEPS) classifica-se como uma proposta de ação estratégica visando contribuir para a transformação e qualificação das práticas em saúde, a organização de ações e dos serviços, os processos formativos e das práticas pedagógicas na formação de profissionais no âmbito da saúde. A efetivação deste núcleo requer um trabalho intersetorial capaz de articular o desenvolvimento individual e institucional, ações, serviços, gestão setorial e atenção à saúde.

Pensando nisso e, partindo de um diagnóstico realizado no Hospital Distrital Dr. José Gomes da Silva, localizado na cidade de Itaporanga - PB, onde foi 
desenvolvida a pesquisa para a construção deste artigo, estabeleceu-se que a Educação Permanente em Saúde se constitui em estratégia para desenvolver os serviços de saúde, pois implica em constantes atualizações por meio de ações e planejamentos intencionados voltadas ao apoio, fortalecimento de conhecimentos, desenvolvimento de habilidades e atitudes que venhas a repercutir no interior das relações da equipe até suas práticas organizacionais, intersetoriais a implicarem nas políticas em que se inserem as ações em saúde.

A Educação Permanente em Saúde ainda pode ser conceituada como algo que acontece no cotidiano das pessoas e das organizações, sendo esta feita a partir dos problemas enfrentados levando em consideração os conhecimentos e as vivências e/ou experiências que as pessoas já trazem consigo, sendo proposto que a educação dos trabalhadores da saúde se faça a partir da problematização estabelecida no processo de trabalho e, considera ainda que as necessidades de formação e desenvolvimento dos trabalhadores sejam pautados pelas necessidades enfrentadas mediante a saúde das pessoas e populações, estando entendida aqui como um processo de aprendizagem-trabalho. Sendo assim, como o Núcleo de Educação Permanente em Saúde, tendo este como uma ferramenta de gestão, irá auxiliar no funcionamento das atividades do Hospital Distrital Dr. José Gomes da Silva, bem como proporcionar mudanças positivas nos índices de saúde do mesmo?

Assim, partindo do diagnóstico da instituição e da análise de situação no setor saúde, situando-o no cenário e no contexto descrito, é de tamanha relevância destacar dimensões e aspectos que poderão contribuir e/ou auxiliar para o desenvolvimento de uma política de educação permanente em saúde. Nesse sentido, objetiva-se identificar a percepção dos funcionários do Hospital Distrital Dr. José Gomes da Silva, localizado no município de Itaporanga - PB, no que diz respeito ao trabalho desenvolvido pelo Núcleo de Educação Permanente em Saúde (NEPs) visando o fortalecimento do trabalho multidisciplinar intersetorial a partir de prioridades estabelecidas na construção do Plano de Ação da instituição de saúde. Com isso, para obter resultados eficazes faz-se necessário priorizar atividades para os gestores do sistema, bem como envolvimento e comprometimento por parte do conjunto de trabalhadores e não apenas de suas lideranças.

Aqui, a sistemática adotada para a construção desta pesquisa foi de apontar a problematização interna, identificando os problemas comuns e posteriormente apresentando soluções no que tange a questão de Educação Permanente em 
Saúde. Logo, como metodologia, foi utilizada a pesquisa ação e, também, exploratória sendo coletados os dados através de um estudo de campo e pesquisas bibliográficas realizadas em sites e outros estudos encontrados, seguido de uma abordagem qualitativa tendo os funcionários do próprio hospital como colaboradores.

Contudo, este projeto de pesquisa foi elaborado com a finalidade de conduzir os trabalhos a serem desenvolvidos no que tange a educação permanente em saúde dos trabalhadores e usuários de serviço, objetivando identificar a percepção dos funcionários do Hospital Distrital Dr. José Gomes da Silva no que diz respeito ao trabalho desenvolvido pelo Núcleo de Educação Permanente em Saúde (NEPs) tendo este como ferramenta de gestão, visando o fortalecimento do trabalho multidisciplinar intersetorial a partir de prioridades estabelecidas na construção do Plano de Ação da instituição de saúde.

Esta pesquisa foi desenvolvida no Hospital Distrital Dr. José Gomes da Silva, localizado no município de Itaporanga, Estado da Paraíba, tendo como colaboradores os próprios funcionários da instituição. Logo, trata-se de uma pesquisa de cunho exploratório-descritivo, fazendo uma análise interpretativa de modo documental, buscando informações em documentos, sendo estes fontes primárias.

A priori, foram realizadas conversas formais e análise documental sobre 0 NEPS, sua implantação e funcionamento na referida instituição de saúde, isto juntamente com a coordenadora do núcleo e a gestora do hospital. Destarte, ainda com o apoio dos funcionários citados, foi realizado um levantamento dos atendimentos, número de funcionários, leitos e estrutura da instituição e, logo após, convidou-se os funcionários e partiu-se para a construção do Plano de Ação sendo elencados pontos a serem discutidos e trabalhados como prioridades para o melhor desenvolvimento da equipe, tendo em vista o trabalho multidisciplinar e intersetorial.

\section{Núcleo de educação permanente no brasil}

Há mais de duas décadas, o Brasil vem redefinindo a estrutura e o perfil do sistema de saúde. Nos anos noventa os papéis e funções dos entes federados foram reformulados e este novo modelo foi organizado de forma regionalizada, seguindo as diretrizes da descentralização administrativa e operacional, do 
atendimento integral à saúde e da participação da comunidade visando o controle social.

Assim, a formação dos trabalhadores é considerada por Lima (2014) como um dos suportes do Sistema único de Saúde (SUS). Logo, seguindo essa perspectiva, o Brasil instituiu a Política Nacional de Educação Permanente em Saúde (PNEPS) como estratégia e ferramenta de formação e desenvolvimento de trabalhadores da saúde, por meio da Portaria GM/MS n 198/2004 (CUNHA, 2009).

A Política Nacional de Educação Permanente em Saúde (EPS) lançada pelo Ministério da Saúde através da Portaria 198, de fevereiro de 2004, permite identificar as necessidades de formação e de desenvolvimento dos trabalhadores da área da saúde e a construção de estratégias e processos que possa qualificar a atenção e a gestão em saúde, fortalecendo o controle social, tendo este o objetivo de causar um impacto positivo no que diz respeito a saúde individual e coletiva da população. (CAROTTA, KAWAMURA e SALAZAR, 2009).

A Educação Permanente em Saúde (EPS) Faz relação com ensino, serviço, docência e saúde como um conceito pedagógico vindo a contribuir para o aperfeiçoamento do profissional, da gestão e o controle social (LEMOS, 2009). A EPS está alicerçada nos pressupostos da aprendizagem significativa que se torna orientação para as ações do desenvolvimento profissional e das estratégias no processo de mudança das práticas em saúde.

Assim, a mesma apresenta-se como uma estratégia de educação em saúde que tem um olhar voltado para as necessidades e prioridades da população, o que se configura como um processo de gestão, sendo esta participativa e transformadora, incluindo instituições de ensino, trabalhadores, gestores e usuários, formando o que é chamado de "quadrilátero da formação" (PESSÔA et al., 2013).

A EPS tem sido compreendida, quanto as suas práticas metodológicas, como uma estratégia transformadora das práticas realizadas no âmbito da saúde, cuja vem colaborando para o rompimento do paradigma tradicional que norteia os processos de formação dos trabalhadores da saúde. Constitui-se como um instrumento eficaz no que diz respeito ao desenvolvimento pessoal e social do profissional estando baseada nos processos de ensino-aprendizagem, sendo o sujeito que aprende um agente ativo, participativo e gestor de sua própria educação (FRANÇA et al., 2017). 
Na Educação Permanente em Saúde, formação e gestão estão associadas. Dessa forma, Ceccim (2005) nos traz que o conceito de educação permanente em saúde foi pensado para promover a transformação da rede pública de saúde em uma rede de ensino-aprendizagem no exercício do trabalho, possibilitando a relação de congruência entre formação e gestão.

A educação permanente em saúde precisa ser entendida, ao mesmo tempo, como uma prática de ensino-aprendizagem e como uma política de educação na saúde. Ela se parece com muitas vertentes brasileiras da educação popular em saúde e compartilha muitos de seus conceitos, mas enquanto a educação popular tem em vista a cidadania a educação permanente tem em vista o trabalho (CECCIM; FERLA, 2005).

A Política Nacional de Educação Permanente (PNEPS) teve, em seu processo de implementação, a criação de muitos programas com o objetivo de adequar a formação e qualificar os profissionais de saúde a exercerem suas respectivas funções; esses programas foram considerados desarticulados ou fragmentados. Desta forma, tiveram limitada capacidade de produzir impacto sobre as instituições formadoras e, consequentemente, promover alterações nas práticas dominantes no sistema de saúde (MINISTÉRIO DA SAÚDE, 2004).

Uma década após a implantação da política foram identificadas descontinuidades, mudanças de enfoque e questões de financiamento. Tais aspectos parecem dificultar a sua implantação (PINTO, 2014). Outro limite é que as ações de educação podem se mostrar subsumidas nas atribuições da gestão de $\mathrm{RH}$, pois há gestores que não discutem e/ou desconhecem as políticas de gestão da educação na saúde (PIERANTONI, 2012).

No setor da saúde, Educação Permanente é um conceito pedagógico utilizado para expressar as relações entre ensino e ações e serviços, articulando docência e atenção à saúde. A Política Nacional de Educação Permanente em Saúde (PNEPS) ampliou esse conceito, que passou a designar também as relações entre a formação e a gestão setorial, desenvolvimento institucional e controle social em saúde (BRASIL, 2007).

A Política de EPS aponta o fortalecimento da gestão participativa e da responsabilidade compartilhada, com dispositivos que ampliem os espaços para 0 exercício do diálogo, integração, participação, troca de experiências e de conhecimentos e a busca de respostas e soluções coletivas para problemas que 
impedem a atenção integral e de qualidade. Ao mesmo tempo estimula a formação e o desenvolvimento de profissionais que atendam às necessidades dos serviços públicos, a partir de interesses e prioridades identificados pelos próprios sujeitos envolvidos na saúde (SARRETA, 2009).

\section{Núcleo de educação permanente em saúde do hospital distrital dr. José Gomes da Silva}

Diante da realidade de trabalho vivenciada no ambiente hospitalar, onde a sobrecarga de trabalho nos impede de promover atividades educativas, com a construção de saberes e associação da vivência prática e o conhecimento teórico, há a necessidade de promover a educação permanente com os profissionais, não somente com o objetivo de qualificação, mas como meio de integração e motivação dos profissionais (ARAÚJO, 2015).

O Hospital Distrital de Itaporanga Dr. José Gomes da Silva ${ }^{1}$ é referência para quatorze (14) municípios da Paraíba, no Vale do Piancó, nos quais podem ser citados: Boa Ventura, Diamante, Curral Velho, Ibiara, Pedra Branca, Santana de Mangueira, São José de Caiana, Serra Grande, Aguiar, Conceição, Nova Olinda, Santa Inês, Igaracy e Itaporanga, onde está localizado, sendo de grande relevância para essa região, contando com uma equipe de 209 funcionários, tendo a Secretaria de Estado da Saúde como suporte financeiro e técnico. Com isso, o mesmo assiste cerca de pouco mais de 3 mil (três mil) atendimentos mensais, sendo estes de urgência e emergência, adultos e infantis, clínica médica e cirúrgica, obstetrícia e pediátrica.

Em constante processo de construção, o SUS traz em sua essência um grande desafio no processo de garantir o exercício de seus princípios doutrinários a Universalidade, a Integralidade e a Equidade - envolvendo as entidades formadoras, gestores, trabalhadores e usuários num processo contínuo de formação e reflexão, buscando aproximá-los das necessidades de toda a população (MIYAMOTO, 2014).

Atualmente a qualidade na assistência à saúde e a humanização dos serviços têm ocupado espaço na agenda da gestão municipal como meta prioritária para a consolidação do Sistema Único de Saúde (SUS). A EPS trabalha com ferramentas

\footnotetext{
${ }^{1}$ Todos os Dados coletados sobre o Hospital Distrital Dr. José Gomes da Silva, localizado no Município de Itaporanga - PB, foram pesquisados a partir de documentos no/do próprio hospital.
} 
que buscam a reflexão crítica sobre as práticas de atenção, gestão e formação, sendo, por si só, um processo educativo aplicado ao trabalho, possibilitando mudanças nas relações, nos processos, nos atos de saúde e nas pessoas e uma melhor articulação para dentro e para fora das instituições (CAROTTA, KAWAMURA e SALAZAR, 2009).

Para tanto, objetivando cumprir o estabelecido na Portaria 1.996 de 20 de agosto de 2007, sobre a implementação da Política Nacional de Educação Permanente em Saúde, a Comissão Interna do Núcleo de Educação Permanente, resguardada pela Portaria 005/2017 de 01 de Dezembro de 2017, reuniu-se para discutir as problematizações do serviço, posteriormente estabelecerem estratégias de ação para intervenção nos problemas elencados. Neste sentido foi elaborado um plano de ação com a finalidade de conduzir os trabalhos a serem desenvolvidos no que tange a educação permanente em saúde dos funcionários e usuários do serviço.

O Núcleo de Educação Permanente do Hospital Distrital de Itaporanga Dr. José Gomes da Silva, vem contribuindo desde a sua formação até os dias atuais, através da portaria interna de 02/2019, para a melhoria da assistência e dos processos de trabalho, por meio de etapas que possam problematizar a realidade, transformando as práticas profissionais, melhorando permanentemente a qualidade da assistência/cuidados a saúde.

Atualmente, as atividades educativas desenvolvidas são direcionadas para todo corpo de profissionais que atuam no serviço, atuando como órgão de assessoria e subordinação a direção geral do serviço, tendo autonomia plena de decidir sobre assuntos, traçando metas que estiverem relacionadas com as ações de educação permanente, através de um plano de ação anual, onde se faz um levantamento das necessidades e demandas de oferta de formação e qualificação profissional, com vista a melhoria do processo de trabalho, fortalecendo junto com a equipe multidisciplinar, ações de educação, capacitação e desenvolvimento de atividades, visando sempre a melhoria de atendimento e atualização profissional, apoiando os profissionais de saúde do serviço em seu processo de trabalho assistencial no monitoramento e avaliação das ações de saúde e educação permanente.

Assim, sendo o Núcleo de Educação Permanente em Saúde é uma estratégia utilizada pela gestão do Hospital Distrital de Itaporanga, visando trabalhar com a 
perspectiva de construir nos espaços da instituição a capacidade de pensar de forma articulada, qualificando as ações profissionais e o desenvolvimento dos gestores, das equipes de saúde e dos agentes sociais, buscando promover a atenção integral à população com qualidade e resolubilidade, articulando as redes de serviços e fortalecendo a autonomia das pessoas no cuidado a saúde.

Os trabalhos a serem desenvolvidos a partir deste plano foram elencados por ordem de prioridades, discutindo com os técnicos que fizeram parte da construção do mesmo, porém com flexibilidade para possíveis alterações se assim forem necessárias conforme adequação das necessidades regionais. A equipe priorizou da seguinte maneira:

\section{Tabela 1: Sistema de Informação}

\begin{tabular}{|c|c|}
\hline PROBELMAS COMUNS & $\begin{array}{l}\text { Dados não fidedignos; } \\
\text { Incompatibilidade de dados; } \\
\text { Falta de capacitação para os geradores de informação. }\end{array}$ \\
\hline PROPOSTA & $\begin{array}{l}\text { Oferecer orientação do preenchimento dos dados aos digitadores e } \\
\text { funcionários que trabalham na recepção. }\end{array}$ \\
\hline OBJETIVO & Melhorar a qualidade dos dados fornecidos. \\
\hline PÚBLICO ALVO & Recepcionistas e Digitadores. \\
\hline ESTRATÉGIAS & $\begin{array}{l}\text { Prática com exercícios de preenchimento de informação dos dados } \\
\text { e sua devidas atualizações. }\end{array}$ \\
\hline META & 100\% do público alvo \\
\hline PRAZO/PERIODICIDADE & 2018/2019 - Semestral. \\
\hline RESULTADOS & $\begin{array}{l}\text { Ter profissionais preparados para alimentar os sistemas de } \\
\text { informações de modo a obter dados confiáveis e verdadeiros, } \\
\text { servindo de instrumento de planejamento para o serviço. }\end{array}$ \\
\hline
\end{tabular}

Os funcionários que trabalham na parte de repasse das informações apresentam um problema crucial no que diz respeito a veracidade das informações geradas, sendo levantadas dúvidas e a falta de atualização de informações no sistema traz malefícios para o funcionamento do serviço. Assim, faz-se necessário prepará-los e orientá-los quanto aos dados alimentados no sistema.

\section{Tabela 2: Capacitação para Médicos, Enfermeiros e Técnicos de Enfermagem}




\begin{tabular}{|c|c|}
\hline & Falta de preparo no atendimento de emergências. \\
\hline PROPOSTA & $\begin{array}{l}\text { Capacitação para os Enfermeiros e Técnicos de } \\
\text { Enfermagem no que diz respeito a humanização no } \\
\text { atendimento do serviço; }\end{array}$ \\
\hline OBJETIVO & $\begin{array}{l}\text { Capacitar os Enfermeiros e Técnicos para melhorar } \\
\text { o desempenho nos atendimentos; } \\
\text { Qualificar a equipe para o primeiro atendimento de } \\
\text { urgência e emergência, considerando que os } \\
\text { profissionais que atendem os pacientes em } \\
\text { situação de urgência e emergência não possuem } \\
\text { preparação. }\end{array}$ \\
\hline PÚBLICO ALVO & Médicos, Enfermeiros e Técnicos de Enfermagem. \\
\hline ESTRATÉGIAS & $\begin{array}{l}\text { Oficinas, Palestras e atividades práticas voltadas } \\
\text { para o atendimento de urgência e emergência e } \\
\text { humanização; }\end{array}$ \\
\hline META & $100 \%$ do público alvo \\
\hline PRAZO/PERIODICIDADE & 2018/2019 - Anual. \\
\hline RESULTADOS & $\begin{array}{l}\text { Ter profissionais preparados para o atendimento de } \\
\text { urgência e emergência na intervenção de situações } \\
\text { de risco diminuindo assim o impacto negativo no } \\
\text { índice de saúde da instituição. }\end{array}$ \\
\hline
\end{tabular}

Capacitar os profissionais de saúde deste serviço para melhoria no desempenho dos atendimentos, qualificando a equipe para os primeiros atendimentos, levando em consideração que todos os profissionais atendem os pacientes em situação de urgência e emergência podem não possuir uma devida preparação.

\section{Tabela 3: Parto Humanizado}

\begin{tabular}{|c|c|}
\hline PROBLEMAS MAIS COMUNS & $\begin{array}{l}\text { Ausência de PPP - Pré-Parto, Puerpério e Pós } \\
\text { Parto; } \\
\text { Falta de equipamentos; } \\
\text { Falta de espaço físico. }\end{array}$ \\
\hline PROPOSTA & $\begin{array}{l}\text { Qualificação, modernização e modificação do } \\
\text { espaço; } \\
\text { Separar sala de pré parto e pós parto; } \\
\text { Implantação de um pré-parto; } \\
\text { Parto Humanizado. }\end{array}$ \\
\hline OBJETIVO & $\begin{array}{l}\text { Valorizar o ambiente com organização de espaço } \\
\text { de trabalho acolhedor. } \\
\text { Possibilitar um melhor atendimento as } \\
\text { gestantes/usuários do serviço. }\end{array}$ \\
\hline PÚBLICO ALVO & Médicos, Enfermeiros e Técnicos de Enfermagem. \\
\hline ESTRATÉGIA & Capacitações, Oficinas e Palestras. \\
\hline META & $100 \%$ do Público Alvo \\
\hline PRAZO/PERIODICIDADE & 2018/2019 - Anual \\
\hline RESULTADO & $\begin{array}{l}\text { Equipe preparada para o atendimento do parto } \\
\text { humanizado. }\end{array}$ \\
\hline
\end{tabular}


A assistência de qualidade ao recém nascido no momento e depois do parto garante um começo de vida saudável, assim, a valorização do ambiente com melhor organização do espaço de trabalho acolhedor possibilitará a mãe, ao bebê e acompanhante ficarem juntos no quarto ou na enfermaria, tendo assim um melhor atendimento as gestantes/usuários do serviço.

A partir de um diagnóstico geral realizado pela equipe, foram dadas como prioridades as questões a cima citadas. Sendo assim, espera-se que sejam seguidas de forma a melhorar o atendimento e bem estar dos usuários do serviço da instituição de saúde.

\section{Considerações finais}

O Hospital Distrital Dr. José Gomes da Silva tem um papel de grande relevância para o município de Itaporanga - PB, sendo ele referência para quatorze municípios e fazendo pouco mais de três mil atendimentos mensais.

A proposta de Educação Permanente em Saúde veio ao encontro das necessidades de aperfeiçoar estes mecanismos, tornando-os mais participativos, respeitando o conhecimento dos profissionais e ampliando os espaços de aprendizagem no próprio local de trabalho. Um dos fatores mais importantes desta proposta é a reflexão crítica dos processos de trabalho. $\mathrm{Na}$ avaliação dos participantes das rodas, a fala que mais se repete refere-se ao espaço propiciado para encontro da equipe e possibilidade de falar sobre os problemas, nós críticos construindo soluções coletivamente. $O$ ato da assistência e as relações melhoram a partir da responsabilização de cada envolvido.

Em resposta às necessidades do Hospital Distrital de Itaporanga, elencadas neste plano conclui-se pela intervenção no que diz respeito a profissionalização dos funcionários, bem como para gestão, tendo entre seus compromissos a formação de parcerias, a participação efetiva dos funcionários nas atividades propostas pelo Núcleo de Educação Permanente (NEP) proporcionando o estímulo da instituição voltado para o fortalecimento das atividades focadas na gestão estratégica.

Assim, almeja-se que a Gestão da Educação Permanente em Saúde, representada pelo NEP, consiga estabelecer as relações de trabalho a partir de uma concepção na qual a participação do funcionário seja fundamental para a efetividade e eficiência do funcionamento do serviço. Dessa forma, o trabalhador é percebido 
como sujeito e agente transformador de seu ambiente e não apenas um mero recurso humano realizador de tarefas previamente estabelecidas pela Gestão.

Nesta perspectiva, o trabalho é visto como um processo de trocas, de criatividade, participação e responsabilização, de enriquecimento e comprometimento mútuos. Essa política pressupõe a garantia de requisitos básicos para a valorização do trabalhador da saúde, assim como a articulação com as necessidades da atenção integral à saúde, visando o fortalecimento do trabalho multidisciplinar e intersetorial.

O Núcleo de Educação Permanente deverá prosseguir com as ações iniciadas no processo de formação dos facilitadores de educação permanente e realizar um Plano Municipal de Educação Permanente, seguindo as orientações e diretrizes propostas no Pacto pela Saúde, assinado pelo Município, Estado e União em outubro de 2007.

\section{Referências}

ARAÚJO, K. Q. M. A. de. Núcleo de Educação Permanente como ferramenta de gestão organizacional no Hospital Universitário Ana Bezerra: um plano de ação técnico aplicado.

Dissertação (Mestrado) - UFPB/CE/CCSA. João Pessoa, 2015.

BRASIL. Portaria N . 1.996, de 20 de agosto de 2007. Dispõe sobre as diretrizes para a implementação da Política Nacional de Educação Permanente em Saúde e dá outras providências. Diário Oficial da União. Brasília, n. 162, 22 ago. Seção 1. 2007.

CAROTTA, F.; KAWAMURA, D.; SALAZAR, J. Educação Permanente em Saúde: uma estratégia de gestão para pensar, refletir e construir práticas educativas e processos de trabalhos. Saúde e Sociedade, v.18, supl.1, 2009.

CECCIM, R.; B. Educação Permanente em Saúde: descentralização e disseminação de capacidade pedagógica na saúde. Ciência e Saúde Coletiva, v. 10, 2005, p. 975-86.

CUNHA, I. S. Educação permanente em saúde e planejamento estratégico situacional: o caso da Secretaria Estadual de Saúde do Piauí [dissertação]. Rio de Janeiro: Fundação Oswaldo Cruz; 2009.

FERLA, A. A. Notas Cartográficas sobre a escuta e a escrita: contribuição à educação das práticas de saúde. In: PINHEIRO, R.; MATTOS, R. A. Construção Social da Demanda: direito à saúde, trabalho em equipe, participação e espaços públicos. Rio de Janeiro, 2005.

FRANÇA, T.; MEDEIROS, K. R. de; BELISARIO, S. A., GARCIA; A. C., PINTO; I. C. de M.; CASTRO, J. L. de; PIERANTONI, C. R. Política de Educação Permanente em Saúde no 
Brasil: a contribuição das Comissões Permanentes de Integração Ensino-Serviço. Ciência \& Saúde Coletiva, 22(6):1817-1828, 2017.

LEMOS, M.; FONTOURA, M.; A integração da educação e trabalho na saúde e a Política de Educação Permanente em Saúde do SUS-BA. Rev Baiana Saúd Púb 2009; 33(1):113-120.

LIMA, S. A. V.; ALBUQUERQUE, P. C.; WENCESLAU, L. D. Educação permanente em saúde segundo os profissionais da Gestão de Recife, Pernambuco. Trab. Educ. Saúde 2014; 12(2):425-441.

MINISTÉRIO DA SAÚDE. Secretaria de Gestão do Trabalho e da Educação na Saúde. Política de educação e desenvolvimento para o SUS: caminhos para a educação permanente em saúde - polos de educação permanente em saúde. Brasília; 2004.

MIYAMOTO, G. A.; Núcleo de Educação Permanente em região de municípios de pequeno / médio porte: desafios e potencialidades, SP. Dissertação (mestrado) - Programa de Mestrado Profissional em Saúde Coletiva da Coordenadoria de Recursos Humanos da Secretaria de Estado da Saúde de São Paulo, 2014, 85p.

PESSÔA, L. R.; FERLA, A. A; ANDRADE J; MENEGHEL S, LINGER, C. A; KASTRUP, E. A educação permanente e a cooperação internacional em saúde: um olhar sobre a experiência de fortalecimento da rede haitiana de vigilância, pesquisa e educação em saúde, no âmbito do projeto Tripartite Brasil-Haiti-Cuba. Divulg Saúd em Debat 2013; 49:165171.

PIERANTONI, C. R; GARCIA, A. C. P. A gestão do trabalho e da educação na saúde em secretarias estaduais e municipais de saúde. Divulg. Saúde Debate 2012; 47:44-54.

PINTO, H. A; SOUSA, A.; FERLA, A. A. Atenção Básica e Educação Permanente em Saúde: cenário apontado pelo Programa Nacional de Melhoria do Acesso e da Qualidade da Atenção Básica (PMAQ-AB). Divulg. Saúde Debate 2014; 51:145-158.

SARRETA, F. O. Educação permanente e saúde para os trabalhadores do SUS. Editora UNESP, São Paulo: Cultura Acadêmica; 2009. Available from SciELO Books $<$ http://books.scielo.org>.

SECRETARIA DE ESTADO DA SAÚDE DE SANTA CATARINA. CIES - Comissão de Integração Ensino e Serviço, Meio-Oeste. Plano Regional de Educação Permanente em Saúde, AMMOC E AMPLASC, 2012. 